\title{
Viscoelastometer for Measurement of Flow and Elastic Recovery
}

\author{
Richard J. Overberg and Herbert Leaderman
}

\author{
(October 3, 1960)
}

\begin{abstract}
An account is given of the design, performance, and method of operation of a concentric cylinder rotational viscoelastometer. The instrument is designed for measurement of flow and time-dependent elastic recovery of viscous elastic liquids for temperatures at which the viscosity lies in the range of $10^{6}$ to $10^{12}$ poises and the elastic recovery is to be studied over a range of time from 1 second to 1 day. It is also suitable for measurement of non-Newtonian flow when the apparent viscosity is not much less than the Newtonian viscosity. The stator is a 1.5 inch precision-bore glass tube, which permits visual observation of the sample. Interchangeable rotors (radius ratios $0.21,0.58,0.92$ ) are provided, the smallest for materials which are only slightly non-Newtonian and have a small compliance. Torque is applied by weights through a torque drum. Mirrors on the rotor shaft and a photoelectric recorder permit continuous recording of the rotor position. Mercury slip rings permit monitoring of rotor temperature. The maximum torque is $5 \times 10^{7} \mathrm{dyne}-\mathrm{cm}$, while the torque due to static friction is $2.5 \times 10^{4}$ dyne-cm. The maximum surface shear stress is $6.5 \times 10^{6}$ dyne-cm ${ }^{-2}$. Temperatures from -70 to $+160{ }^{\circ} \mathrm{C}$ have been realized. "End-effect" studies have been made. Instrument compliance, temperature gradient correction, and the effect of inertia and friction on elastic recovery measurements are discussed. Data reducticn methods for nonlinear behavior are developed.
\end{abstract}

\section{Introduction}

This paper gives an account of the design, performance, and method of operation of a concentric cylinder rotational viscoelastometer for measurement of flow and time-dependent elastic recovery of viscous elastic liquids. The design is based upon that of an earlier simpler instrument which has been previously described $[1] ;{ }^{1}$ the present instrument possesses higher precision, greater experimental convenience, and more versatility than the previous viscoelastometer.

The instrument is designed in the first place to measure linear viscoelastic behavior of low molecular weight polymers, and also of high molecular weight polymers in the rubber-liquid transition region, for the temperature range in which the viscosity lies in the range $10^{6}$ to $10^{12}$ poises. This represents roughly the range of temperature for which the time scale of elastic recovery lies in the range of 1 sec to 1 day. The viscoelastic behavior of other materials such as certain asphalts can also be investigated. Finally, the instrument can be used for the study of nonlinear elastic and flow behavior over a limited range.

\section{Design Principles}

The principles used in the design of the instrument and the relation of this method of measuring linear viscoelastic behavior to other methods will be briefly reviewed. It is well-known that noncrosslinked amorphous polymers above the glass-transition manifest a flow behavior which in general is non-Newtonian. Such materials are also viscoelas-

\footnotetext{
1 Figures in brackets indicate the literature references at the end of this paper.
}

tic, that is, they possess elastic as well as flow properties. Under conditions where the energy storage and the rate of energy dissipation are small, the response to shear stress is linear. The linear viscoelastic behavior in shear of a material may be characterized completely by its behavior in a test with a specified stress history. The most important methods of specification are the response to sinusoidal shear stress or shear strain history (dynamic tests) and the response to step-function shear stress or strain history (creep and stress relaxation tests). In the latter case the shear stress or shear strain is increased stepwise from zero to a given value and maintained constant thereafter. Other stress histories that have been considered are ramp function histories, in which the shear stress or strain is proportional to time [2], and impulse histories in which the stress or strain history varies with time as a delta function [3].

In the first two types of test, the significant range of time or frequency at any given temperature for noncrosslinked amorphous polymers may be of the order of fifteen decades. The practical time or frequency range of any piece of apparatus ranges from about two decades for dynamic tests to a maximum of about six decades for step-function experiments. It appears for such materials that the major effect of change in temperature is to change the time or frequency scale; hence the effective range of a piece of apparatus can be extended by change in temperature of test. The limits of this range depend upon instrumental factors.

From the phenomenological theory of linear viscoelastic behavior it can be shown that in principle the response over the whole significant range of time (or frequency) for any given stress (or strain) 
history can be related to the response in any other type of test with another specified stress (or strain) history [4]. In practice, rheological data that can be measured directly by one method can be calculated from data obtained by another method only if the latter data are of extremely high precision. For example, the viscosity $\eta$ and the steady state compliance $J_{e}$ can be calculated from dynamic data or from stress relaxation data $[5,6]$; however, such calculated values are often unreliable. These quantities on the other hand can be measured very simply with the rotational viscometer. Since the measurement of the limiting value of the elastic deformation for noncrosslinked polymers involves large permanent deformations, a simple shear apparatus in which the shear stress is uniform is not suitable, but a rotational method is indicated.

Considerations of the ranges in viscosity and time lead to a design in which the material is contained in a rotationally symmetrical surface with vertical axis and closed bottom. The material is sheared by the relative rotation between the cup and another rotationally symmetric, coaxial surface immersed in the material. Of the various possible designs, that using circular cylinders was chosen to permit accurate construction and alinement of the two surfaces. The Navier-Stokes equation for this geometry cannot be solved; it is necessary to use the solution for infinitely long cylinders together with an empirically determined end-correction. This "endeffect" has been obtained experimentally for ranges of two of the three geometrical parameters. The procedures for obtaining the elastic part of the shear creep compliance, $J(t)-t / \eta$, from elastic recovery measurements over a range of time in a rotational viscometer have been given previously [8].

In the present arrangement, the outer surface (the "cup") is fixed. The arrangement is such that a constant torque can be rapidly applied to or released from the inner cylinder (the "rotor"). Thus the shear stress on any given particle of fluid increases stepwise in time to a constant value and subsequently decreases similarly to zero. A study has been made of the frictional torque in the rotor system. The lower limit of the range in torque for a series of measurements of viscoelastic behavior is chosen to be well above this frictional torque.

In general, the flow and elastic behavior of viscoelastic liquids are found to be nonlinear. The steady flow of a polymeric material manifesting nonNewtonian behavior is associated with normal stress phenomena; in the concentric cylinder arrangement secondary flow will take place, manifesting itself as a rising up of the liquid in contact with the inner cylinder, the so-called Weissenberg effect, together with complicated axial and radial flow [9]. In measurements made with the viscoelastometer the upper limiting value of the torque is such that there is no perceptible disturbance to the free surface. It is then assumed that, over the whole range of torque, liquid particles move essentially in circles around the axis of the instrument and that secondary flow is negligible. Under these circum- stances it is often found that the ratio of angular velocity to torque increases linearly with torque. A procedure was given previously for obtaining from such data the relationship between shear stress and rate of shear in a simple shear test $[1,8]$. In this paper a more general method due to Pawlowski [10] is given.

The method of Pawlowski has been extended to derive the retarded elastic recovery behavior in simple shear for both finite and vanishing shear stress from nonlinear recovery data obtained in the viscometer.

The lower limiting value of the shear creep compliance $J_{g}$ of amorphous polvmers is of the order of $10^{-10} \mathrm{~cm}^{2} /$ dyne. However, the lowest measurable compliance is determined by the instrument compliance, which is limited by the necessity of reducing the moment of inertia of the rotor system to the minimum. The instrument compliance has been measured using a liquid cooled below its glass transition.

As a result of thermal conduction along the rotor shaft, the temperature of the rotor is slightly different from that of the thermostat. A slight temperature gradient thus exists through the liquid. A method has been developed, based upon relationships relating time-scale and temperature, to correct for this temperature gradient.

It has been demonstrated that the elastic part of the shear creep compliance and the non-Newtonian flow of noncrosslinked amorphous polymers are closely correlated [11]; these quantities cover a wide range of values depending upon the molecular weight distribution. The sensitivity of the present apparatus is varied by using interchangeable rotors of three different diameters. The sensitivity range can be further extended by the use of extra length rotors and stators. Since it is necessary to observe the free surface for manifestation of the Weissenberg effect, precision bore glass tubes are used for stators. This arrangement has the added advantage of allowing direct measurement of depth of immersion, which can vary appreciably over the range of temperatures of observation especially when the clearance between the plane ends is large and the radial gap is small.

\section{Description of the Apparatus}

The general arrangement of the viscoelastometer is shown diagrammatically in figure 1. An interchangeable rotor $A$ is connected to the Kovar-glass insulating link $D$, which in turn is attached to the rotor shaft $K$. Two ball bearings $J$ locate the rotor shaft in the housing $V$, which is supported by the collar $E$ through a spacing ring $H$ and gage blocks $I$. The spacing ring $H$ is machined to a thickness corresponding to $0.025 \mathrm{in}$. clearance between the plane ends of the rotor and stator in the absence of the gage blocks. This clearance may be increased in steps to one inch, by means of gage blocks. The collar $E$ rests on the support ring $F$ which is located on the upper platform $X$. The stator $B$, a precision 


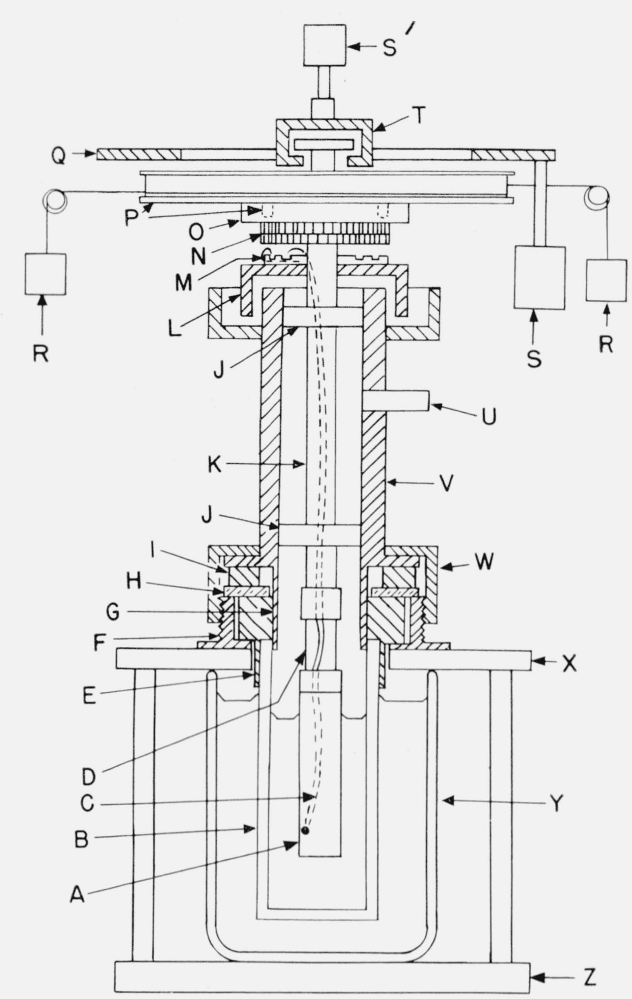

Figure 1. Section drawing of viscoelastometer.

A, Interchangeable rotor; B, Precision-bore glass stator; C, Rotor thermocouple; D, Kovar-glass insulating link; E, Collar; F, Support ring; G, Stator centering sleeve; H, Spacing ring; I, Gauge blocks; J, Axial thrust ball bearings; K, Rotor shaft; L, Mercury seal; M, Mercury slip-rings for thermocouple contacts; N, Multiple mirror; $\mathrm{O}$. Torque drum adapter; $\mathrm{P}$. Torque drum; Q, Torque drum arresting ring; R, Weights; S, S', Solenoids; T, Drum removal device; U, Gas inlet tube; V, Rotor shaft housing: W, Locking ring: $\mathrm{X}$, Upper platform; $\mathrm{Y}$, Dewar vessel; $Z$, Sub-base.

bore glass tube with fused or cemented plane end, is cemented to the metal collar $E$. This is alined coaxially with the rotor by the centering sleeve $G$, which is an extension of the shaft housing. The rotor shaft housing, spacing ring, gage blocks, and stator are clamped together by the locking ring $W$. The shaft housing $V$ carries a mercury seal $L$ and a gas inlet tube $U$, which may be used to maintain the sample in the stator under an inert atmosphere, or, removing the mercury from $L$, to flush the bearings $J$ with an inert gas. The leads from a thermocouple $C$ in the rotor terminate in mercury slip rings $M$, attached to the rotating portion of the mercury seal. The upper end of the rotor shaft carries a multiple mirror system $N$, which is part of the recording arrangement and an adapter $O$, for the detachable torque drum $P$, which transmits its torque through four pins. The drum arresting ring $Q$ can be pulled down against the torque drum by means of solenoids $S$ to prevent rotation of the rotor system to which the weights $R$ are attached. Two equal loads apply a pure torque to the drum by means of cords as shown. A solenoid $S^{\prime}$ activates a drum removal device $T$, which serves rapidly to disengage the drum $P$ from the adapter $O$, and to press the drum against the arresting ring $Q$.

The stator is surrounded by a constant temperature bath contained in an unsilvered Dewar vessel $Y$.
The controlled bath temperature does not vary more than $\pm 0.02{ }^{\circ} \mathrm{C}$. Experiments have been carried out over the temperature range -40 to $160{ }^{\circ} \mathrm{C}$. The lowest bath temperature that has been reached is $-70{ }^{\circ} \mathrm{C}$. The rotor and bath temperature can be monitored continuously during a test, and the rotor thermocouple serves to indicate when the rotor has come to temperature equilibrium following a change in bath temperature. Platinum foil is used for both the stationary and rotating contacts in the mercury rings. The rotor and bath thermocouples, placed side by side in a bath, give the same indicated emf over a range of temperature. Rotation of the rotor causes no change in the emf indicated. The temperatures are measured by means of a potentiometer sensitive to about $2 \mu \mathrm{v}$.

A photograph of the viscometer is shown in figure 2. Disassembly is accomplished by lowering the subbase $Z$, seen figures 1 and 2 . After the central part of the subbase has been removed the Dewar may be lowered through the table. This arrangement allows the stator tube to be removed from below. At this stage the rotor can be removed from the connecting link, which extends below the platform.

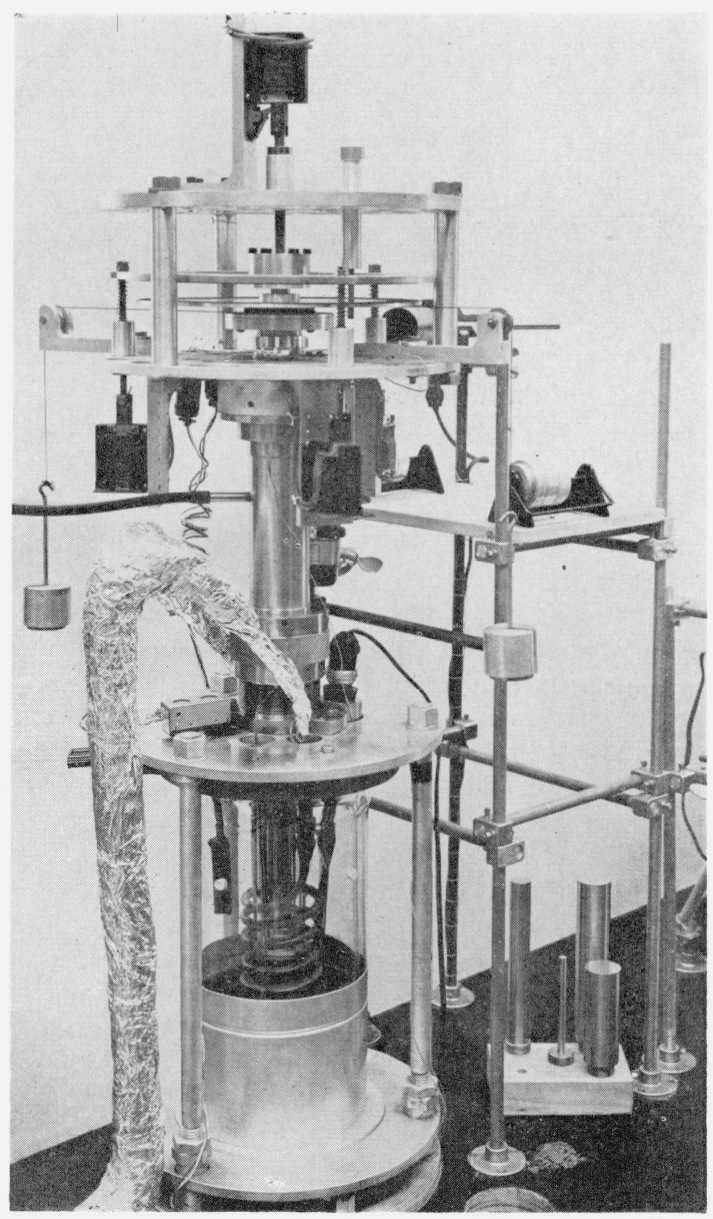

FIGURE 2. Photograph of viscoelastometer. 


\subsection{Rotor and Stator System}

Neglecting the effect of the end, the shear stress at the surface of the rotor $\sigma_{1}$ is equal to the torque divided by twice the immersed volume of the rotor and the shear stress on any given cylindrical element varies inversely as the square of the radius. For materials that are only slightly non-Newtonian and for which the steady state elastic compliance $J_{e}$ is small, the sensitivity of the apparatus is increased by using a small-diameter rotor, and conversely. The set of five interchangeable rotors, of diameters respectively $0.79 \mathrm{~cm}, 2.22 \mathrm{~cm}$, and $3.50 \mathrm{~cm}$, are shown in figure 3 , which also shows the long and

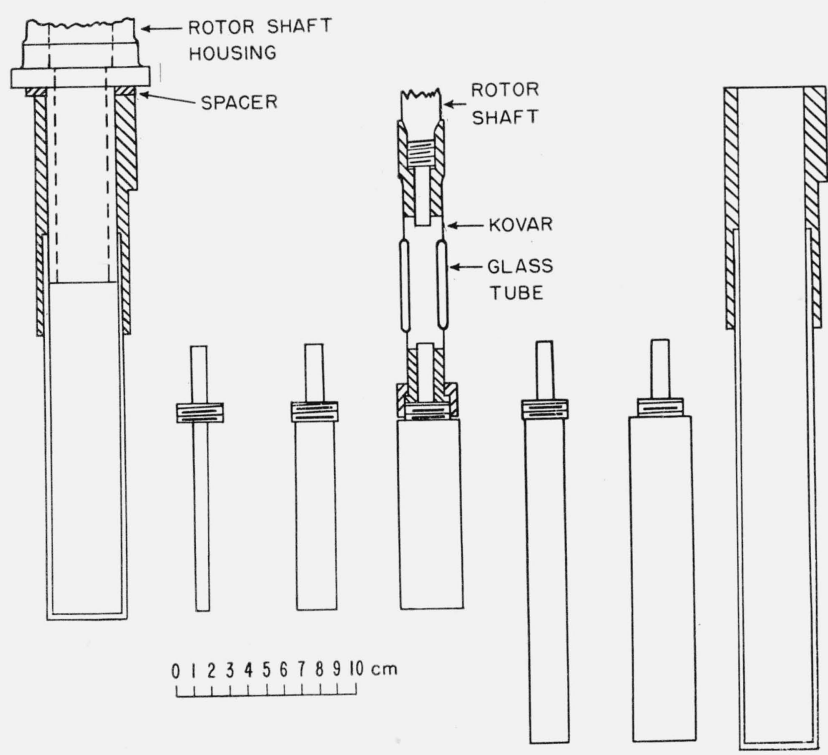

Figure 3. Rotor and stator systems.

The drawings represent to scale the five rotors, two sizes of stator tubes, and thermal insulating link.

short stators to be used respectively with the two long and three short rotors. The stator tubes are of $3.81 \mathrm{~cm}$ internal diameter and $0.32 \mathrm{~cm}$ nominal wall thickness, and the steel collars are cold-cemented in a jig by means of an acid proof cement. ${ }^{2}$

The steel collars of the stators are provided with three splines subtending and angle of $45^{\circ}$ to the axis of rotation; these rest on similar splines in the support ring referred to previously. In order to remove the stator tube it is merely necessary first to loosen the clamp $W$ seen in figure 1, which presses the collar of the shaft housing against the stator collar. The rotor system is raised slightly; the stator is rotated so that the two sets of splines clear each other, and then the stator is lowered through the platform $Z$. Shown in figure 3 is the Kovar-glass insulating link and the method of attachment of the link to the rotor and to the lower end of the rotor shaft.

2 Sauereisen Acid Proof Cement No. 31, Sauereisen Cement Company, Pittsburgh, $\mathrm{Pa}$.
Table 1 gives the range in the shear stress at the surface of the rotor $\sigma_{1}$, and at the surface of the stator $\sigma_{0}$, for the three rotor diameters. The range in values of torque $W$ is from the maximum that has been used, to a minimum taken arbitrarily at about 30 times the friction torque. The depths of immersion in table 1 are reasonable values for the two lengths of rotor.

TABLE 1.-Values of shear stress at rotor and stator surfaces

\begin{tabular}{|c|c|c|c|c|}
\hline Torque $W$ & $\begin{array}{l}\text { Rotor diam- } \\
\text { eter } 2 \mathrm{a}_{1}\end{array}$ & $\begin{array}{l}\text { Depth of im- } \\
\text { mersion } h\end{array}$ & $\begin{array}{l}\text { Shear stress at } \\
\text { rotor surface } \sigma_{1}\end{array}$ & $\begin{array}{l}\text { Shear stress at } \\
\text { stator surface } \sigma 0\end{array}$ \\
\hline $\begin{array}{r}\text { dyne-cm } \\
8 \times 10^{z} \\
\text { to } \\
4 \times 10^{7}\end{array}$ & $\begin{array}{l}\mathrm{cm} \\
\quad 3.50\end{array}$ & cm & $\left\{\begin{array}{c}\text { dyne/cm² } \\
2.8 \times 10^{3} \\
\text { to } \\
1.4 \times 10^{5}\end{array}\right.$ & $\begin{array}{l}\text { dyne/cm } \\
2.4 \times 10^{3} \\
\text { to } \\
1.2 \times 10^{5}\end{array}$ \\
\hline $\begin{array}{l}8 \times 10^{5} \\
\text { to } \\
4 \times 10^{7}\end{array}$ & 2. 22 & 7.5 & $\begin{array}{l}1.4 \times 10^{4} \\
\text { to } \\
6.9 \times 10^{5}\end{array}$ & $\begin{array}{l}\text { 4. } 7 \times 10^{3} \\
\text { to } \\
2.3 \times 10^{5}\end{array}$ \\
\hline $\begin{array}{c}8 \times 10^{5} \\
\text { to } \\
4 \times 10^{7}\end{array}$ & 0. 794 & 7.5 & $\begin{array}{c}\text { 1. } 09 \times 10^{5} \\
\text { t3 } \\
5.4 \times 10^{6}\end{array}$ & $\begin{array}{l}\text { 4. } 7 \times 10^{3} \\
\text { to } \\
2.3 \times 10^{4}\end{array}$ \\
\hline
\end{tabular}

The sensitivity of the apparatus may be characterized by the ratio of angular rotation of the rotor system per unit torque to compliance of the material. If $J_{e}$ is the steady-state elastic compliance and if $\theta_{r}$ is the maximum recovery angle in radians, then

$$
\frac{\theta_{r}}{W J_{e}}=\frac{1 / c^{2}-1}{4 \pi h a_{0}^{2}}
$$

where $h$ and $a_{0}$ are the depth of immersion and the stator radius, and $c$ is the ratio of the rotor diameter to stator diameter. The numerator of the righthand side ranges from 22 (for the smallest rotor) to 0.2 . The practicable range of values of $J_{e}$ may be estimated by taking reasonable values of $\theta_{r}$ and $h$ equal to 0.2 radian and $7.5 \mathrm{~cm}$, respectively. In table 2 this range is obtained from eq (1) for the largest diameter rotor with the lower limit of the torque in table 2 and for the smallest diameter rotor with the upper limit of torque. From table 2 it is seen that the instrument is suitable for measurement of values of $J_{e}$ in the range $10^{-4} \mathrm{~cm}^{2} /$ dyne to $10^{-7}$ $\mathrm{cm}^{2} /$ dyne.

Fiduciary marks in the form of circles are scribed on the cylindrical portion of each rotor and are located at exactly $8.89 \mathrm{~cm}$ and $16.193 \mathrm{~cm}$ from the plane base for the short and long rotor respectively. These marks are required in the case of the long rotors and are helpful with the short rotors for the measurement of depth of immersion, which is accomplished by means of a traveling microscope.

\subsection{Torque Application and Release}

Details of the arrangement for applying and releasing a constant torque are shown in figure 4 . The three lower solenoids in figure 2 actuate the drumarresting ring $\mathrm{C}$ in figure 4 ; coil springs normally hold this ring against upper stops. The solenoids are first energized by closing the switch $F$. The push-button 


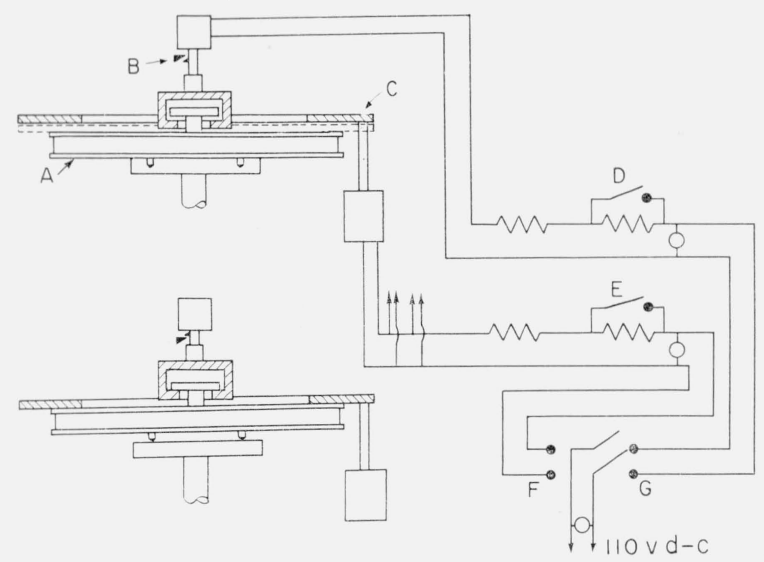

Figure 4. Torque drum arrest and removal device. A, Torque drum; B, Catch; C, Drum arresting ring; D, E, F, G, Switches.

$E$ provides a momentary overload which pulls the ring $C$ against the springs into contact with the torque drum $A$; the weights are then hung from the cords. The contacting surfaces are provided with friction materials, so that the pressure of the ring $C$ on the drum $A$ prevents rotation of the drum. When the switch $\mathrm{F}$ is opened, the ring $\mathrm{C}$ is returned by the springs as shown in the upper diagram in figure 4, and the drum is thus released. When it is desired to remove the torque, the upper solenoid is similarly energized by the switch $G$ in figure 4 . When the overload button $D$ is pressed momentarily, the drum $A$ is pulled off the shaft, and held against the ring $C$ as shown in the lower diagram in figure 4 ; the friction surfaces again prevent rotation of the torque drum. The rotor system is now free from torque, and retarded elastic recovery can take place. After the weights are removed, the upper solenoid is deenergized. The catch $B$ in figure 3 holds the drum clear of the rotor. The frame carrying the load application and release mechanism is supported on a flange resting on the stationary cup of the mercury seal. This frame together with the torque drum can be easily removed after opening the connectors in the solenoid leads.

The ten-inch diameter torque drum is interchangeable with a drum of 2.5 in. diameter; the necessity for the latter drum can be shown by the following considerations. It has previously been shown that in order to obtain the limiting value of $J(t)-t / \eta$, it is necessary for the preceding flow test to be of duration of the order of $10^{-3} \eta$ seconds for low molecular weight polyisobutylene [1]. The same coefficient applies to a series of blends of polyisobutylene fractions in the same molecular weight range [11], and also to a high molecular weight polyisobutylene of the order of one million molecular weight. Thus, if $R$ is the drum radius, $W$ the torque and $h$ the depth of immersion, the distance $\delta$ descended by the weights to reach the steady state is given approximately by:

$$
\delta=\frac{10^{-3} W R}{\pi h}\left[\frac{1}{4 a_{1}^{2}}-\frac{1}{4 a_{0}^{2}}\right]
$$

where $2 a_{1}$ and $2 a_{0}$ are the rotor and stator diameters respectively. Taking $h$ equal to $7.5 \mathrm{~cm}$ and $W$ equal to the minimum practicable torque of $8 \times 10^{5}$ dyne$\mathrm{cm}$, it is found from eq (2) that for the 10-in. diameter drum, $\delta$ is equal to $650 \mathrm{~cm}, 60 \mathrm{~cm}$, and $5 \mathrm{~cm}$, for the small, intermediate, and large diameter rotors, respectively. The maximum range in $\delta$ is 120 $\mathrm{cm}$; thus only a small range in values of $W$ is possible for the intermediate size rotor. For the smaller drum the above values of $\delta$ are divided by four, and hence a wider range of values of $W$ becomes available for the intermediate size rotor. In order to obtain a range in values of $W$ for the smallest rotor, an even smaller drum and possibly a considerably smaller stator tube would be required.

\subsection{Autographic Recording System}

The recording system is shown diagrammatically in figure 5. The angular position of the rotor as a function of time is traced on the chart of a slightly modified photoelectric recorder. ${ }^{3}$ On the rotor shaft

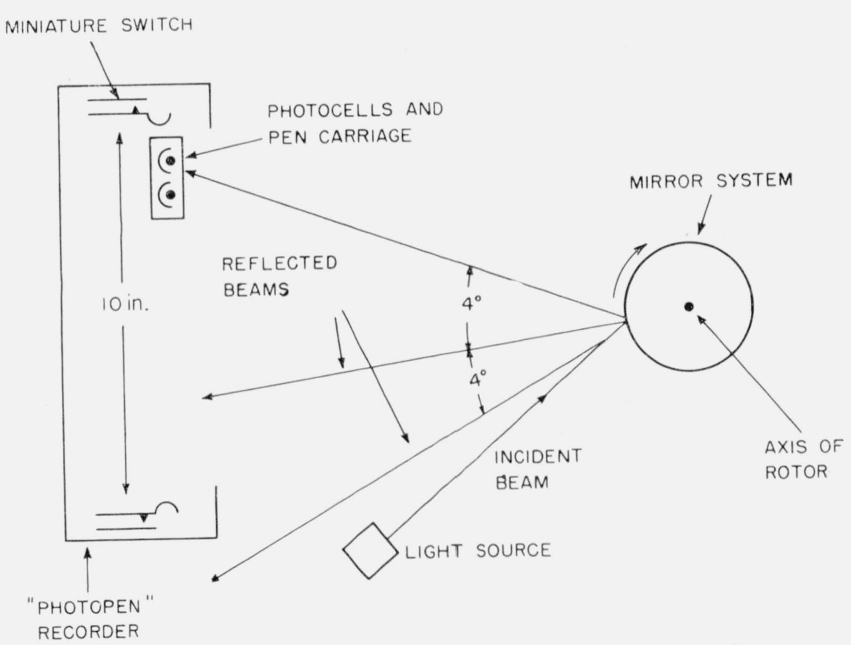

Figure 5. Diagrammatic view of optical recording system.

are two banks of mirrors; each bank comprises 45 plane, plated, and polished surfaces of a brass block, normals to adjacent surfaces subtending an angle of $8^{\circ}$ with each other. The two banks are staggered symmetrically to each other. The mirrors serve to reflect the incident light beam from an adjacent light source on to the pair of photocells on the pen carriage of the recorder. The distance of the recorder from the viscometer is such that $4^{\circ}$ rotation of the rotor corresponds to nearly the full chart width of ten inches. This arrangement permits continuous recording of the angular displacement of the rotor.

3 Beckman "Photopen”. National Technical Laboratories, South Pasadena, Calif. 
The recorder has been modified so that a continuous record of flow or recovery can be obtained with either continuous or intermittent chart drive. The continuous record is obtained by means of miniature switches at each end of the pen-carriage travel. When the carriage actuates the appropriate miniature switch, the light source is momentarily extinguished, and the pen carriage is caused to travel back until it picks up the next beam. The time for total return of the carriage is about two seconds, thus even if transfer from one beam to the next takes place during the initial rapid recovery, little or no extrapolation is required.

It is desirable, following the initial rapid recovery, to slowdown the speed of the chart, which travels normally at $1 \mathrm{in}$. per minute. This is done by an intermittent chart drive, which moves the chart for $1 \mathrm{~min}$. in each hour. The device consists of three cams driven at 1 revolution per hour by a synchronous motor. By means of miniature switches the cams switch on and off in the appropriate sequence the light source, servoamplifier of the recorder, and chart drive. The changeover from continuous to intermittent chart drive can be preset by means of a timing clock. This clock, together with the reversal switch for controlling the direction of continuous recording and the switches for application and removal of torque, are mounted in the recorder stand.

Calibration of the recording system was made by measuring the distance travelled by the pen carriage of the recorder when moved from 1 reflected light beam to the adjacent beam, with the rotor stationary. This was done for a total of 97 successive displacements, the rotor system being moved by hand. In this way $360^{\circ}$ rotation of the rotor was found to correspond to a total pen-carriage displacement of 778.77 in., thus 1 in. displacement on the chart was equivalent to $0.462^{\circ}$ rotation of the rotor. Large angular velocities were obtained from measurement in the chart travel direction of the distance between alternate traces, corresponding to reflections from adjacent mirrors on the same bank, thus giving the time for a nominal rotation of $8^{\circ}$. In the previous measurements the sum of successive pairs of displacements was found to vary from 17.22 to $17.45 \mathrm{in}$., with a mean of $17.31 \mathrm{in}$. There is seen to be no necessity for the two banks of mirrors to be staggered precisely at $4^{\circ}$ to each other.

The recording system displayed no measurable drift over several hours, thus justifying measurements of elastic recovery over extended periods of time with a balanced photocell arrangement.

\section{Performance}

Measurements of the friction of the rotor system, end effect calibration, and corrections for instrument compliance and temperature gradient in the test material will be discussed.

\subsection{Instrument Friction}

In figure 6 are shown some measurements of angu- lar velocity $\Omega$ as a function of torque $W$ using a Newtonian liquid, namely, commercial hydroabietyl alcohol, which has a viscosity of about $10^{6}$ poises at $12{ }^{\circ} \mathrm{C}$. It is seen that for all cases, the angular velocity is proportional to $\left(W-W_{f}\right)$, where $W_{f}$ is the friction torque equal to about $2.5 \times 10^{4}$ dyne-cm, and is independent of temperature, depth of immersion $h$, rotor diameter $2 a_{1}$, and end clearance $d$. The circles, squares, and diamonds in figure 6 show the effect of varying respectively the rotor diameter, depth of immersion, and temperature. Thus in measurements of flow behavior, the instrument friction is simply accounted for by taking the effective torque as $\left(W-W_{f}\right)$. The effect of friction on elastic recovery measurements is more complicated and is considered in section 7 .

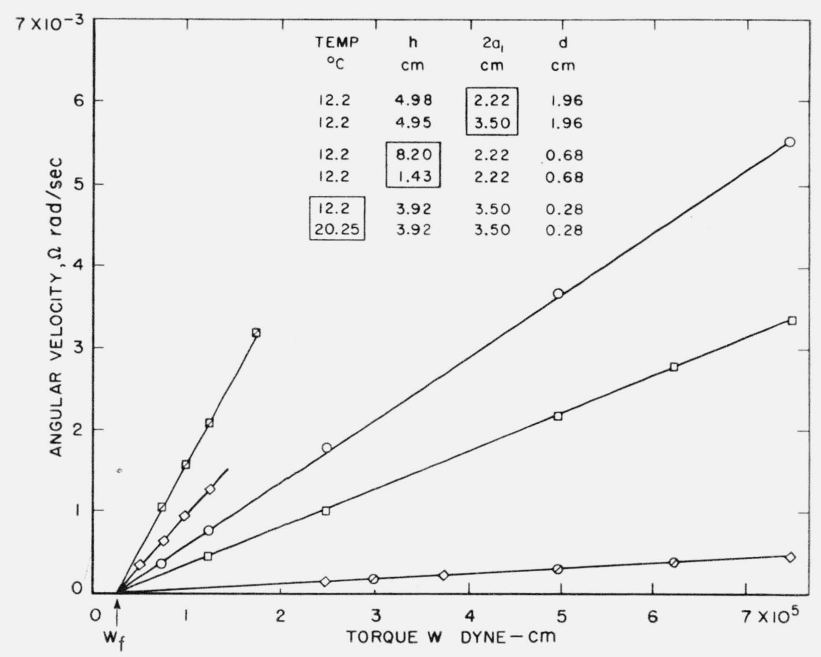

Figure 6. Angular velocity versus torque; $h$, depth of immersion; $2 \mathrm{a}_{1}$, rotor diameter; and $\mathrm{d}$, end clearance.

\subsection{Instrument Constant and End-Effect}

It is desired to obtain the instrument constant for the steady flow of a Newtonian liquid in the viscometer. This instrument constant $K$ may be defined as the ratio of $\Omega \eta$ to effective torque $\left(W-W_{f}\right)$ for any given geometry, where $\eta$ is the viscosity of the liquid. The same instrument constant is applicable for a material manifesting linear viscoelastic behavior, provided that inertia effects can be ignored [8].

The viscoelastometer has been operated with a pool of mercury in the base of the stator, the surface of the mercury extending above the plane surface of the rotor. The test material is floating on the mercury and is located entirely in the annular space between the cylindrical surfaces of the stator and rotor. Under these circumstances the motion of the particles in the test liquid is the same as in a portion of a Couette-type viscometer of infinite length. For this arrangement the instrument constant is then given by: 


$$
K=\frac{\Omega \eta}{W-W_{f}}=\frac{1-c^{2}}{4 \pi a_{1}^{2} h}
$$

In eq (3) it is, of course, assumed that the torque due to the mercury is negligible compared to that due to the test liquid.

More usually, the test liquid occupies the space between the plane ends of the rotor and stator, together with the adjoining portion of the annular space between the cylindrical surfaces. Under these circumstances, the torque on the cylindrical portion is modified, and there exists a torque on the plane base of the rotor. For this arrangement it is reasonable to assume that the instrument constant is given by:

$$
K=\frac{\Omega \eta}{W-W_{f}}=\frac{1-c^{2}}{4 \pi a_{1}^{3}\left(\frac{h}{a_{1}}+\frac{\Delta h}{a_{1}}\right)}
$$

where $\Delta h / a_{1}$ is independent of $h / a_{1}$ for sufficiently large values of the latter ratio; $\Delta h / a_{1}$ thus is a function of two independent geometrical parameters such as $c$ and $d / a_{1}$, where $d$ is the distance between the plane ends of the rotor and stator.

The justification for this assumption for a particular geometry is given by the data in figure 7 , which shows plots of $\left(W-W_{f}\right) / \Omega$ against $h$ for the intermediate diameter rotor $\left(2 a_{1}=2.22 \mathrm{~cm}\right)$ for different values of end clearance, using hydroabietyl alcohol at $12.2{ }^{\circ} \mathrm{C}$ as the test liquid. The data for five values of $d$ are fitted statistically to a series of straight lines of the same slope. The results with the smallest end clearance, represented by the broken line in figure 7 , were erratic and were not used in calculating the intercepts and best slope.

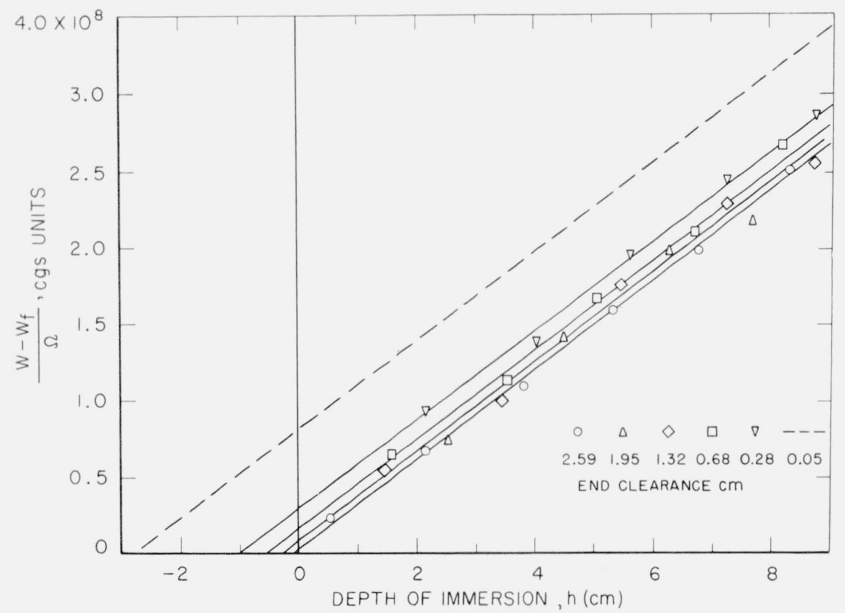

Figure 7. (Effective torque)/(angular velocity) versus depth of immersion for several values of end clearance.

The "end effect" $\Delta h$ is the negative of the intercept on the abscissa.
The results of the end-effect measurements are shown nondimensionally in figure 8 , where the ratios of end effect to rotor diameter, $\Delta h / 2 a_{1}$, are plotted as circles against the ratios of end clearance to rotor diameter. The points appear to fall on a rectangular hyperbola; this is demonstrated by the plot also given in figure 8 of $2 a_{1} / \Delta h$ against $d / 2 a_{1}$, in which the results of the observations are represented as squares; the points are seen to fall on a straight line passing through the origin. A crude theory of end effect can be proposed, in which it is assumed that the torque on the cylindrical portion of the rotor is the same as on the same length of infinite rotor, and that the torque on the plane base is the same as that on a circle of the same diameter which forms part of an infinite plate in a rotating disk viscometer. This theory predicts that $2 a_{1} / \Delta h$ is proportional to $d / 2 a_{1}$, as shown by the broken line in figure 8 . The end effect is seen to be much greater than that predicted by this crude theory.

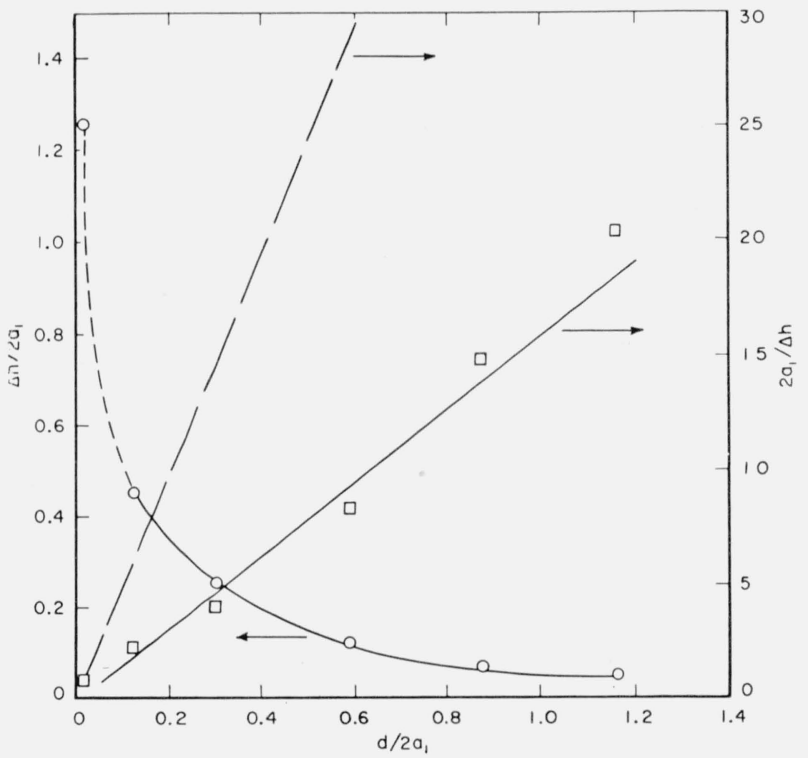

FIGURE 8, End effect measurements $\bigcirc, \Delta \mathrm{h} / 2 \mathrm{a}_{1}$ versus d $/ 2 \mathrm{a}_{1}$; $\square, 2 \mathrm{a}_{1} / \Delta \mathrm{h}$ versus $\mathrm{d} / 2 \mathrm{a}_{1}$. The broken straight line represents the results of an elementary theory.

Similar measurements have been made using the large rotor. However, the plot of $\left(W-W_{f}\right) / \Omega$ against $h$ gave very erratic data from which no conclusions could be drawn.

The only significant data in the literature relevant to this end-effect study are due to Wadleigh [12]. For a value of c equal to 0.290 , these results agree with the empirical relationships:

$$
\frac{2 a_{1}}{\Delta h}=0.64+0.275 \frac{d}{2 a_{1}}
$$

for a range of values of $d / 2 a_{1}$ from unity to four. 


\subsection{Instrument Compliance}

The instrument compliance has been measured using hydroabietyl alcohol, cooled below its glass transition, as the test liquid. The rotation of the mirror system as measured by displacement of the trace on the recorder chart was observed for removal of a given torque on the rotor following the usual procedure. The angular rotation was found to be proportional to torque, leading to a compliance of $0.97 \times 10^{-10} \mathrm{radian} / \mathrm{dyne}-\mathrm{cm}$. This includes the contribution of the compliance of the hydroabietyl alcohol. Assuming a value of shear modulus of $10^{10}$ dynes $/ \mathrm{cm}^{2}$ for glassy hydroabietyl alcohol, this contribution is found to be $0.95 \times 10^{-13}$ radian/dyne$\mathrm{cm}$. Hence the apparatus compliance $C$ may be taken as $0.97 \times 10^{-10} \mathrm{radian} /$ dyne-cm. These data were obtained using the large-diameter $(3.50 \mathrm{~cm})$ rotor; it is assumed that the instrument compliance is the same for the intermediate diameter $(2.22 \mathrm{~cm})$ and small $(0.79 \mathrm{~cm})$ rotors.

The correction $\Delta J$ due to instrument compliance to be subtracted from the material shear creep compliance calculated from the observed value of $(\theta / W)$ is

$$
\Delta J=\frac{\pi(h+\Delta h) C}{\left(\frac{1}{4 a_{1}^{2}}-\frac{1}{4 a_{0}^{2}}\right)}=\frac{C}{K} .
$$

For example, assuming $(h+\Delta h)$ equal to $7.5 \mathrm{~cm}$, then for the intermediate diameter rotor $\left(2 a_{1}=2.22 \mathrm{~cm}\right)$, $\Delta J$ is equal to $1.7 \times 10^{-8} \mathrm{~cm}^{2} /$ dyne; for the small diameter rotor $(0.79 \mathrm{~cm}), \Delta J$ is equal to $1.5 \times 10^{-9}$ $\mathrm{cm}^{2} /$ dyne.

Thus even with the smallest rotor, it is not possible to measure the lower limiting value $J_{g}$ of the shear creep compliance of an amorphous polymer. Since this compliance is of the order of $10^{-10} \mathrm{~cm}^{2} /$ dyne, it would be masked by the instrument compliance. However, it would be possible to measure sucb small values of shear compliance by changing the instrument to an arrangement in which rods of circular or rectangular cross sections are twisted. For example, the compliance of a rod of length $10 \mathrm{~cm}$, diameter $2 \mathrm{~cm}$, and shear modulus $10^{10}$ dynes $/ \mathrm{cm}^{2}$ is $6.4 \times 10^{-10}$ radians/dyne-cm; this is considerably greater than the instrument compliance.

\subsection{Temperature Gradient Correction}

As a result of thermal conductivity along the rotor shaft, the temperature of the rotor is slightly different from that of the thermostat. Measurements of the difference between the rotor temperature $T_{1}$ and the bath temperature $T_{0}$ show that this difference $\Delta \mathbb{T}$ (equal to $T_{0}-T_{1}$ ) varies linearly with bath temperature over a wide range of temperature. Except for natural rubber, there do not appear to exist published data on the thermal conductivity of linear polymers in the liquid state. The thermal con- ductivity of the Pyrex glass of the stator tube is greater by a factor of seven than the thermal conductivity of natural rubber (at room temperature). It is reasonable to assume the thermal conductivities of linear nonpolar polymers in the liquid state are very similar, and theretore that the temperature of the inside wall of the stator is close to that of the bath, for arrangements with the intermediate and small diameter rotors. It is reasonable to assume also that the temperature in the test liquid varies linearly with the radius. If $\Delta T$ is small, then the viscosity of the test liquid varies approximately linearly with radius. With these assumptions, the effect has been calculated of the temperature gradient on steady flow, and also on displacement of the time scale for elastic recovery.

Let $\eta_{1}$ be the viscosity of the liquid at the surface of the rotor, corresponding to temperature $T_{1}$, and let $\eta_{1}\left(1-k^{\prime}\right)$ be the viscosity at the surface of the stator, corresponding to temperature $T_{0}$. Let $\Omega_{0}$ be the angular velocity for a given torque if the temperature is uniform, and the viscosity and temperature are respectively $\eta_{1}\left(1-k^{\prime}\right)$ and $T_{0}$. Let $\Omega_{\mathrm{a}}$ be the angular velocity for the same torque with the temperature gradient. It can then be shown that

$$
\frac{\Omega_{a}}{\Omega_{0}}=1-\frac{k^{\prime} a_{0}}{a_{0}+a_{1}} .
$$

For many polymers the coefficient $k^{\prime}$ can be calculated from known viscosity-temperature relations. Except in the vicinity of the glass transition, the viscosity $\eta$ at temperature $T$ is related to the viscosity $\eta_{1}$ at temperature $T_{1}$ by the expression [13]:

$$
\log \left(\frac{\eta}{\eta_{1}}\right)=A\left(\frac{1}{T^{m}}-\frac{1}{T_{1}^{m}}\right)
$$

Then:

$$
k^{\prime}=2.3 m A \Delta T / T_{1}^{m+1} \text {. }
$$

TABLE 2. Values of $J_{e}$ for elastic recovery of 0.2 radian, with $7.5 \mathrm{~cm}$ depth of immersion

\begin{tabular}{c|c|c}
\hline Torque, $W$ & $\begin{array}{c}\text { Rotor di- } \\
\text { ameter } 2 a_{1}\end{array}$ & $J_{e}$ \\
\cline { 2 - 3 } dyne-cn & $c m$ & $c m^{2} /$ dyne \\
$8 \times 10^{5}$ & 3.50 & $4.5 \times 10^{-4}$ \\
$4 \times 10^{7}$ & 0.79 & $7.8 \times 10^{-5}$ \\
& & \\
\hline
\end{tabular}

From about the glass transition temperature to $100^{\circ} \mathrm{C}$ above this temperature, the viscosity may be represented by a modified form of the Williams-LandelFerry relation $[13,14,15]$ :

$$
\log \left(\frac{\eta_{1}}{\eta}\right)=\frac{900\left(T-T_{1}\right)}{c_{2}\left(c_{2}+T-T_{1}\right)}
$$

where $c_{2}$ is an adjustable parameter. For this case,

$$
k^{\prime}=2.3\left(\frac{900 \Delta T}{c_{2}^{2}}\right) .
$$


Now let $\eta_{a}$ be the apparent viscosity measured in the viscoelastometer with the temperature gradient. Then from eq (6) the viscosity $\eta_{0}$ for an isothermal viscometer at temperature $T_{0}$ is given by:

$$
\left.\begin{array}{c}
\eta_{0}=\eta_{a}\left(1-\frac{k^{\prime} a_{\supset}}{a_{0}+a_{1}}\right) \\
\text { and } \log \eta_{0}=\log \eta_{a}-\frac{k^{\prime} a_{0}}{2.3\left(a_{0}+a_{1}\right)} \cdot
\end{array}\right\}
$$

Let $t_{a}$ be the time to reach a specified angular deformation in a creep or recovery experiment in the viscoelastometer with temperature gradient, and let $t_{0}$ be the time to reach the same deformation in an isothermal viscoelastometer at temperature $T_{0}$. Then

$$
\log t_{0}=\log t_{a}-\frac{k^{\prime} a_{0}}{2.3\left(a_{0}+a_{1}\right)} .
$$

Equations (6), (10), and (11) give the corrections to angular velocity, viscosity, and logarithms of recovery times due to temperature gradient. Thus if $\Delta T$ and therefore $k^{\prime}$ is positive, the logarithmic time scale for elastic recovery corrected to temperature $T_{0}$ is obtained by reducing the observed logarithmic time scale by an amount $k^{\prime} a_{0} / 2.3 \quad\left(a_{0}+a_{1}\right)$.

\section{Method of Operation}

Different filling procedures to obtain bubble-free specimens are used according to whether the material is in the glassy state at room temperature (for example, polystyrene), or is a high molecular weight liquid (for example, polyisobutylene, $M_{w}$ equal to $1.56 \times 10^{6}$, viscosity at $25{ }^{\circ} \mathrm{C}$ about $10^{11}$ poises) or a low molecular weight polymeric liquid (tor example, polyisobutylene, $M_{v}$ equal to $4.1 \times 10^{4}$, viscosity at $25^{\circ} \mathrm{C}$ about $10^{6}$ poises).

In the first case a cylindrical plug is molded, such that the diameter at room temperature is just less than the bore of the stator. The plug is inserted in the bottom of the stator. The whole rotor system is raised so that the tube can be inserted from below as previously described. The Dewar vessel is raised into position from below and the thermostat is set to a temperature corresponding to a viscosity of the polymer in the range $10^{7}$ to $10^{8}$ poises. The rotor system is then released, so that the foot of the rotor presses on the upper surface of the polymer because of the weight of the rotor system. After a period of time of the order of one week, the rotor system has sunk to the limit of its travel, so that its weight is now carried through the gage blocks by the collar at the top of the stator tube. The collar that clamps the stator tube and rotor system to the support ring is finally tightened. A similar procedure has been used in the case of high molecular weight polyisobutylene. In this case an appropriate molding procedure must be used; otherwise air bubbles will appear gradually in the interior of the specimen following removal from the mold.
In the case of a low molecular weight polymer, air is unavoidably entrapped in the material as it is placed on the bottom of the stator. It is therefore heated to reduce its viscosity to $10^{5}$ poises or less, so that the larger air bubbles can rise at a significant rate. When only small air bubbles remain, the rotor system is allowed to sink into position. Tests have been carried out on a low molecular weight polymer in which the stator tube was first filled with an appropriate amount of mercury. When the rotor system was in position, the foot of the rotor was below the mercury surface, and the polymer was in the form of an annulus floating on the mercury.

In carrying out an experiment to measure elastic recovery, the load is applied as discussed earlier. At the appropriate time for load removal the switch reversing the direction of automatic return of the pen carriage is actuated, and the load removed as previously described, just before the pen carriage reaches the limit of its travel. When the recovery has become sufficiently slow, the chart drive is changed from continuous to intermittent motion.

\section{Data Reduction for Nonlinear Behavior}

The shear stress on a cylindrical annulus varies inversely as the square of the radius. In the region of nonlinear behavior, under suitable conditions, secondary flow is negligible, and it is desired to obtain the behavior in simple shear from the flow and recovery behavior of a material in the viscoelastometer.

It has been mentioned previously that in order to avoid the Weissenberg effect, the maximum measurable apparent fluidity is limited to about twice the zero shear fluidity. Over this range it usually appears that $\Omega / W$ varies linearly with $W$. This is consistent with the empirical Ferry flow law

$$
\left(\frac{1}{\eta_{a}}\right)=\left(\frac{1}{\eta_{0}}\right)\left(1+\sigma / G_{i}\right)
$$

where $\left(1 / \eta_{a}\right)$ is the ratio of rate of shear to shear stress $\sigma$ in simple shear, $\left(1 / \eta_{0}\right)$ is the (Newtonian) fluidity, and $G_{i}$ is a constant (for a given material at a given temperature). If we define a Couette viscosity $\eta_{c}$ for a non-Newtonian liquid as that quantity obtained by inserting observed values of $\Omega$ and $\dot{W}$ in the Couette formula, eq (3), with appropriate corrections for $\Omega, W$, and $h$, then:

$$
\left(\frac{1}{\eta_{c}}\right)=\left(\frac{1}{\eta_{0}}\right)\left[1+\frac{\sigma_{1}\left(1+c^{2}\right)}{2 G_{i}}\right] \text {. }
$$

Thus $\eta_{0}$ and $G_{i}$ may be obtained conveniently from a plot of $\left(1 / \eta_{c}\right)$ against $\sigma_{1}$.

In the general case, this plot is nonlinear, and a method due to Pawlowski [10] is suitable for the determination of the flow behavior in simple shear 
using a rotational viscometer, when $c$ is not near unity. Let the rate of shear corresponding to a simple shear stress $\sigma$ be $f(\sigma)$. Then

$$
\Omega=\frac{1}{2} \int_{c^{2} \sigma_{1}}^{\sigma_{1}} \frac{f(\sigma)}{\sigma} d \sigma
$$

where $\sigma_{1}$ and $c^{2} \sigma_{1}$ are the shear stresses at the surface of the rotor and stator walls respectively, and $\Omega$ is the corresponding angular velocity. For a particular value of $\sigma_{1}$ equal to $k$; we obtain from eq (14):

$$
\left.2 \sigma_{1} \frac{d \Omega}{d \sigma_{1}}\right|_{\sigma_{1}=k}=\left[f(\sigma)-f\left(c^{2} \sigma\right)\right]_{\sigma=k} .
$$

If now the torque is reduced in the ratio $c^{2}$, the corresponding angular velocity is given by:

$$
\left.2 \sigma_{1} \frac{d \Omega}{d \sigma_{1}}\right|_{\sigma_{1}=c^{2} k}=\left[f(\sigma)-f\left(c^{2} \sigma\right)\right]_{\sigma=c^{2} k}
$$

Hence

$$
\begin{aligned}
\left.f\left(\sigma_{1}\right)\right|_{\sigma_{1}=k} & =2\left\{\left.\sigma_{1} \frac{d \Omega}{d \sigma_{1}}\right|_{\sigma_{1}=k}\right. \\
& \left.+\left.c^{2} \sigma_{1} \frac{d \Omega}{d \sigma_{1}}\right|_{\sigma_{1}=c^{2 k}}+\cdots\right\}
\end{aligned}
$$

Thus if $\Omega$ is plotted against $\sigma_{1}$, the terms in the brackets on the right hand side are the intercepts $\mathrm{AB}, \mathrm{CD}$, etc., as in figure 9 ; the rate of shear at the surface of the rotor is given by twice the sum of these intercepts.

It is of course necessary to carry the graphical summation only down to a shear stress $k^{*}$, below which the flow behavior is substantially Newtonian. The remainder is then

$$
\left.\frac{2 k^{*}}{1-c^{2}} \frac{d \Omega}{d \sigma_{1}}\right|_{\sigma=k^{*}}
$$

This method can be extended to the case of nonlinear viscoelasticity. Assume that a simple shear stress $\sigma$ is applied at infinite negative time and removed at zero time. Let the decrease in shear strain from zero time to a subsequent time $t$ be $J_{\sigma}$ $(t, \sigma)$. In the case of nonlinear behavior $J(t, \sigma)$, which we will call the recovery compliance, is a function of $\sigma$. If now in a rotational viscometer the shear stress at the surface of the rotor with a given constant torque is $\sigma_{1}$, the angular recovery $\theta\left(t, \sigma_{1}\right)$ at a time $t$ following removal of torque is given by

$$
\theta\left(t, \sigma_{1}\right)=\frac{1}{2} \int_{c^{2} \sigma_{1}}^{\sigma_{1}} J(t, \sigma) d \sigma
$$

The recovery compliance at time $t$ corresponding to a particular value of $\sigma_{1}$ equal to $k$ is given by

$$
\begin{aligned}
\left.J(t, \sigma)\right|_{\sigma=k}=2\left[\left.\frac{d \theta\left(t, \sigma_{1}\right)}{d \sigma_{1}}\right|_{\rho_{1}=k}\right. & \\
& \left.+\left.\frac{c^{2} d \theta\left(t, \sigma_{1}\right)}{d \sigma_{1}}\right|_{\sigma_{1}=c^{2} k}+\cdots\right]
\end{aligned}
$$

Thus the recovery behavior in simple shear is obtained from measurements of angular recovery as a function of $\sigma_{1}$.

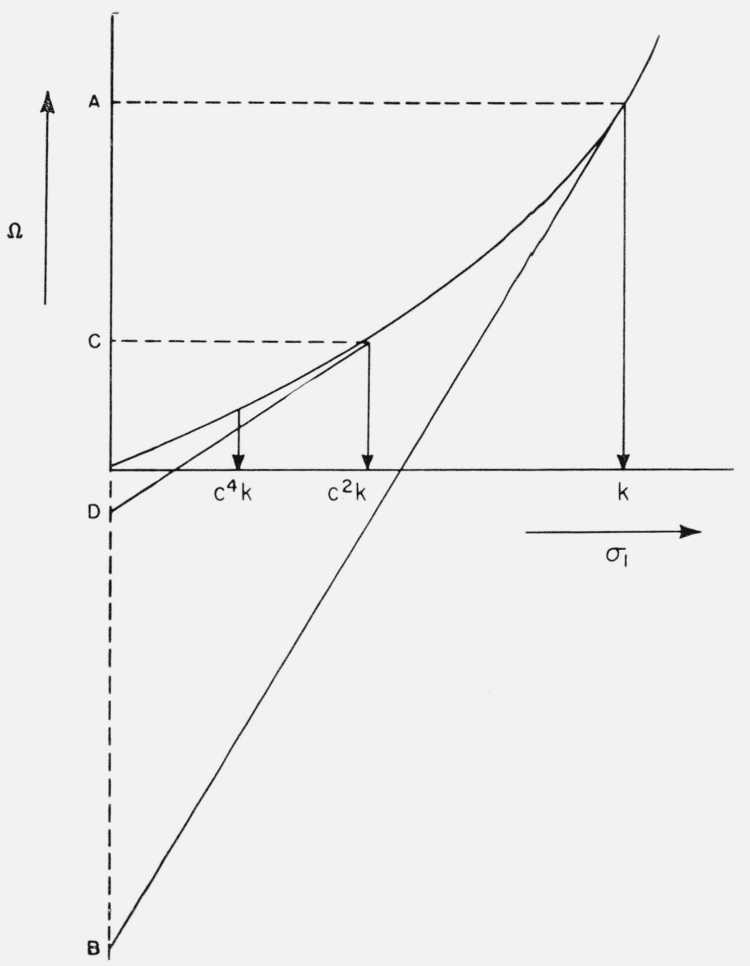

Figure 9. Graphical method of obtaining rate of shear as function of shear stress from plot of angular velocity $\Omega$ versus shear stress $\sigma$ at surface of rotor.

\section{Effects of Inertia and Friction on Recovery}

The effects of the inertia and friction of the rotor system on the observed retarded elastic recovery will now be considered. A dynamic representation of the viscoelastometer system is given in figure 10 (a). For the purpose of the present discussion the springs representing instrument compliance and glass compliance of the material may be omitted, as in figure $10(\mathrm{~b})$. In the models of figure 10 there is represented to a first approximation the viscoelastic behavior of the liquid under conditions where this behavior is linear; a more exact representation is obtained, of course, by adding further Voigt elements with different retardation times $\tau_{i}$. The direction of the frictional torque $W_{f}$ is such as to oppose the deformation of the material. 


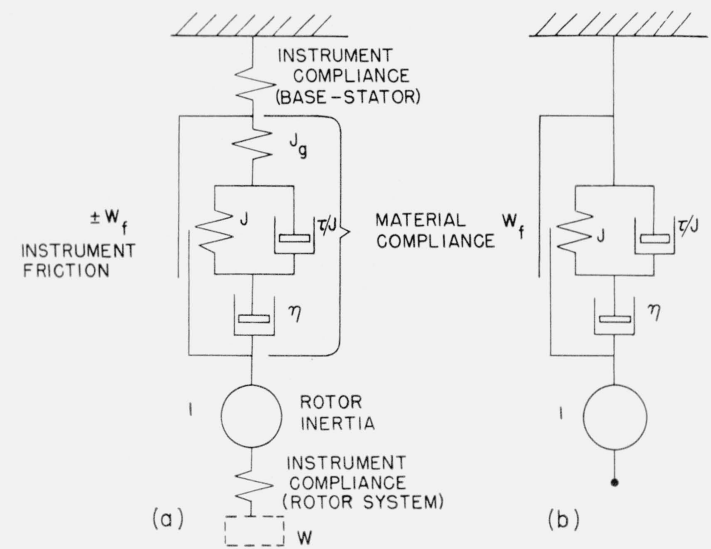

Figure 10. (a) Dynamic representation of viscoelastometer system. (b) Mechanical model for retarded elastic recovery with instrument friction and inertia.

We assume that a constant torque $W$ is first applied to the system until the steady state is reached, and then instantaneously removed. Just before this instant the spring in figure $10(\mathrm{~b})$ is stretched, and the upper dashpot is stationary; the lower dashpot is extending at a uniform rate. On the removal of the external torque $W$ there occurs a transient loading of the material due to rotor inertia. In addition, contraction of the spring results in a load corresponding to the friction torque $W_{f}$, which leads to a steady extension of the lower dashpot. When the rate of contraction of the spring equals the rate of extension of the lower dashpot, contraction of the three-element model representing the material ceases, and relaxation thereafter takes place at constant deformation.

The portion of the record corresponding to removal of load is always "sharp-edged"; there is no perceptible rounding-off due to inertia of the system. The inertia loading on the material can thus be considered as a "spike" of very short duration. The residual elastic deformation of the Voigt element is negligible for times that are long compared to the duration of this spike; there remains to be considered the effect of this transient loading on the flow of the system. This reduces to an apparent material compliance $\Delta J$. Let $I$ be the moment of intertia of the rotor system, and let $-\omega_{1}$ and $\omega_{2}$ be the angular velocities just before and just after removal of torque $W$. It can then be shown that

$$
\Delta J=I\left(\omega_{1}+\omega_{2}\right) / W \eta .
$$

If reasonable values of the parameters are assumed, it appears that $\Delta J$ is negligible for the present viscoelastometer.

In order to estimate the effect of instrument friction on elastic recovery, we will assume as before that the material manifests linear viscoelastic behavior; it can be represented for example by a model consisting of a series of Voigt elements in series with a dashpot. This is loaded by a friction element which exerts a constant torque $W_{f}$ opposing the direction of deformation of the material. Now let $\theta(0)$ represent the angular position of the rotor at the instant of torque removal, and let $\theta(t)$ be the position at a subsequent time $t$. If $W$ is the external torque that is removed and if $J(t)-t / \eta$ is the elastic part of the shear creep compliance, it can be shown that

$$
J(t)-t / \eta=\frac{\theta(0)-\theta(t)}{K\left(W-2 W_{f}\right)}+\left(\frac{W_{f}}{W-2 W_{f}}\right) t / \eta
$$

The second term results from reduction in recovery due to the flow of the material under the friction load. The instrument constant $K$ is given by:

$$
K=\frac{\left(1-c^{2}\right)}{4 \pi a_{1}^{2}(h+\Delta h)}
$$

taking end-effect into account. The previous assumptions lead to cessation of recovery at a time $t$ given approximately by:

$$
\left.L(\tau)\right|_{\tau=\imath} \simeq \frac{W_{f} t}{\left(W-2 W_{f}\right) \eta}
$$

where $\left.L(\tau)\right|_{\tau=t}$ is the value of the retardation spectrum when $\tau$ is equal to $t$. This analysis assumes that the frictional torque for very small values of angular velocity is the same as that measured for more rapid rates of rotation, as in figure 6 . At small angular velocities it appears that $W_{f}$ can be quite variable, which makes the second term in eq (19) somewhat uncertain. The best estimate of the steady state of shear compliance is thus made by evaluating $J(t)-t / \eta$ by means of eq (19) up to the time corresponding to that at which recovery ceases, and then extrapolating to zero slope on a logarithmic time plot.

In the case of nonlinear behavior the calculation of the effect of friction on retarded elastic recovery becomes more difficult. The observed angular recovery $\theta_{0}\left(t, \sigma_{1}\right)$ at a time $t$ following removal of an effective torque $W-W_{f}$ is less than the "ideal" recovery $\theta\left(t, \sigma_{1}\right)$ which would be obtained in the absence of a frictional torque $W_{f}$ opposing the recovery.

If we define a Couette recovery compliance $J_{c}(t$, $\left.\sigma_{1}\right)$ corresponding to recovery in a frictionless viscometer following flow in which the shear stress at the rotor is $\sigma_{1}$ (equivalent to an effective torque $\left.W-W_{f}\right)$, then

$$
J_{c}\left(t, \sigma_{1}\right)=\frac{\theta\left(t, \sigma_{1}\right)}{K\left(W-W_{f}\right)} .
$$

If it is assumed that the reduction in recovery at time $t$ is equal to the angular displacement due to a small torque $W_{f}$ applied at zero time, then

$$
\theta\left(t, \sigma_{1}\right)-\theta_{0}\left(t, \sigma_{1}\right)=W_{f} K J_{r}(t, 0)+W_{f} K t / \eta_{0}
$$

where $J_{r}(t, 0)$ is the elastic part of the shear creep compliance at very small shear stresses, and $\eta_{0}$ is 
the Newtonian viscosity. Combining eqs (22) and (23), we obtain:

$$
\frac{\theta_{0}\left(t, \sigma_{1}\right)+W_{f} K t / \eta_{0}}{K\left(W-2 W_{f}\right)}=\frac{\left(W-W_{f}\right) J_{c}\left(t, \sigma_{1}\right)-W_{f} J_{\tau}(t, 0)}{\left(W-2 W_{f}\right)} .
$$

As $W$ approaches $2 W_{f}$, the right-hand side of eq (24) is seen to approach $J_{\tau}(t, 0)$, thus eq (23) becomes:

$$
\begin{aligned}
\theta\left(t, \sigma_{1}\right)-\theta_{0}\left(t, \sigma_{1}\right)=W_{f} K t / \eta_{0} \\
\quad+W_{f} K \operatorname{Lim}_{W \rightarrow 2 W_{f}}\left[\frac{\theta_{0}\left(t, \sigma_{1}\right)+W_{f} K t / \eta_{0}}{K\left(W-2 W_{f}\right)}\right] .
\end{aligned}
$$

The nonlinear retarded elastic recovery in simple shear is then obtained using eq (17).

Reversal of retarded elastic recovery. According to the theory of linear viscoelasticity, if the viscoelasticity is only of the relaxational type that can be represented by models consisting only of springs and dashpots, then the elastic part of the creep compliance, $J(t)-t / \eta$, is a function increasing monotonically with time, as in figure 11 . This function reaches an asymptotic limit at infinite time; this limit can be assumed to be reached in a frictionless instrument at a finite time which depends upon the sensitivity of the recorder.

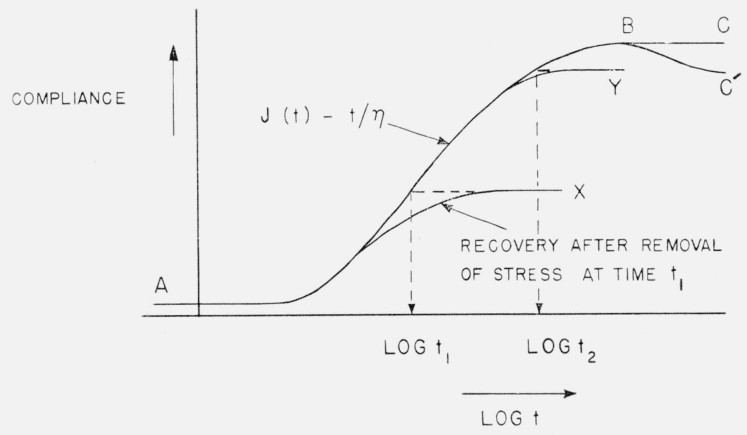

Figure 11. Reversal of retarded elastic recovery.

Let us assume that the point B in figure 11 corresponding to a time $t_{3}$ represents the limit of observability. Then the observable curve is as $\mathrm{ABC}$ where the curve beyond $\mathrm{B}$ is parallel to the abscissa, and $\mathrm{BC}$ represents the apparent asymptote. If recovery observations are made following a time under stress equal or greater than $t_{3}$, then these observations should give the curve $A B C$. If the stress is removed at an earlier time $t_{1}$, in figure 11 , then the recovery curve is as shown by $\mathrm{AX}$. The total recovery is equal to the elastic component of the deformation at time $t_{1}$. If the stress is removed at a later time $t_{2}$, then the elastic component corresponding to the asympotote of the curve $\mathrm{AX}$ is increased. It is found that if the time under load in the rotational viscometer is sufficiently long, the recovery curve does not tend to an observationally limited asymptote $\mathrm{BC}$, but shows a reversal of the direction of creep recovery as represented by $\mathrm{BC}^{\prime}$.
This behavior has been observed in a specimen of polystyrene under conditions where the flow and early part of the recovery were linear with torque; the effect became much more marked with increase in torque. It has been observed in a specimen of polyisobutylene in which the foot of the rotor was immersed in mercury. Thus this phenomenon cannot be accounted for by nonlinearity in viscoelastic behavior, by instrument friction, or by secondary flow in the region of the plane ends.

This effect has been previously observed in measurements of the shear creep and recovery in a rotational viscoelastometer on a specimen of asphalt [16]. The instrument was a narrow-gap conicylindrical viscometer, and the viscoelastic behavior was linear and nonthixotropic over the range of the reported data. As in our experiments on polystyrene, the amount of reversal in recovery became much greater with increase in shear stress, and the time corresponding to the maximum recovery was reduced with increase in shear stress. The elastic recovery curves on asphalt were obtained by measuring the total recovery as for example at $\mathrm{X}$ or $\mathrm{Y}$ following a time under stress equal to $t_{1}$ or $t_{2}$ in figure 11. Unless one postulates the existence of a nonlinear resonance type viscoelastic behavior superimposed upon a linear relaxation type behavior, no explanation can be given for the existence of this phenomenon observed in the two viscoelastometers with linear polymers and with asphalts. Until this behavior can be accounted for, further uncertainty is added to the interpretation of observed angular recovery data.

\section{Nomenclature}

$J(t)-t / \eta=$ elastic part of shear creep compliance.

$J_{g}=$ glass compliance.

$\sigma_{1}=$ shear stress at surface of inner cylinder (rotor).

$\sigma_{0}=$ shear stress at surface of outer cylinder (stator).

$2 a_{1}=$ rotor diameter.

$h=$ depth of immersion.

$W=$ torque.

$2 a_{0}=$ stator diameter.

$c=$ ratio of diameters $=a_{1} / a_{0}$.

$J_{e}=$ steady-state elastic compliance.

$\theta_{r}=$ total recovery angle.

$\eta=$ viscosity.

$R=$ radius of torque drum.

$d=$ "end clearance" between plane ends of stator and rotor.

$W_{f}=$ friction torque.

$\Omega=$ angular velocity.

$K=$ instrument constant.

$\Delta h=$ "end-effect".

$C=$ apparatus compliance.

$T_{1}=$ rotor temperature.

$T_{0}=$ bath temperature.

$\eta_{1}=$ viscosity at rotor surface.

$\eta_{1}\left(1-k^{\prime}\right)=$ viscosity at stator surface.

$\Omega_{0}=$ angular velocity of isothermal viscometer.

$\Omega_{a}=$ angular velocity of viscometer with temperature gradient.

$1 / \eta_{a}=$ apparent fluidity in simple shear.

$1 / \eta_{0}=$ Newtonian fluidity $(\equiv 1 / \eta)$.

$\eta_{c}=$ Couette viscosity.

$k=$ value of $\sigma_{1}$ for specified torque.

$J(t, \sigma)=$ recovery compliance (in simple shear, stress $\sigma$, recovery time $t$ )

$\theta\left(t, \sigma_{1}\right)=$ angular recovery at time $t$. 


\section{References}

[1] H. Leaderman, R. G. Smith, R. W. Jones, J. Polymer Sci. 14, 47 (1954)

[2] T. L. Smith, J. Polymer Sci. 20, 89 (1956); Trans. Soc. Rheol. 2, 131, (1958).

[3] H. Leaderman, Elastic and creep properties of filamentous materials, (Textile Foundation, Washington, 1943)

[4] See for example:

(a) B. Gross, Theories of viscoelasticity, (Herrmann et Cie., Paris, 1953).

(b) A. J. Staverman, F. Schwarzl, Linear deformation behavior of high polymers. Chapter in H. A. Stuart, ed. Die Physik der Hochpolymeren, vol. IV, (Springer, Berlin, 1956).

(c) H. Leaderman, Viscoelasticity phenomena in amorphoud high polymeric systems, Chapter in F. R. Eirich, ed., Rheology, theory and applications, vol. II (Academic Press, New York, N.Y., 1958).

[5] I. L. Hopkins, R. W. Hamming, J. Appl. Phys. 28, 906 (1957).

[6] K. Ninomiya, J. Coll. Sci. 14, 49 (1959).

[7] See for example:

(a) S. Oka, Principles of rheometry, chapter in F. R Eirich, ed., Rheology, theory and applications, vol. III (Ácademic Press, New York, N.Y., 1960).

(b) G. Barr, Monograph of viscometry, (Oxford, 1931).
[8] H. Leaderman, J. Polymer Sci. 13, 371 (1954).

[9] A. F. H. Ward, P. Lord, Proc. second internat. congress rheology, V. G. W. Harrison, ed., (Butterworth, London, 1954)

[10] J. Pawlowski, Koll. Z. 130, 129 (1953).

[11] H. Leaderman, R. G. Smith, L. C. Williams, J. Polymer Sci. 36, 233 (1959).

[12] W. H. Wadleigh, BS J. Research 11, 65 (1933) RP577.

[13] See for example:

T. G. Fox, S. Gratch, S. Loshaek, Chapter viscosity relationships for polymers in rheology, theory and applications vol. I, F. R. Eirich, ed., (Academic Press, New York, N.Y., 1956).

[14] M. L. Williams, R. F. Landel, J. D. Ferry, J. Am. Chem. Soc. 78, 3701 (1955)

[15] H. Leaderman, Ann. Rev. Phys. Chem. 9, 179 (1958).

[16] R. N. J. Saal, J. W. A. Labout, J. Phys. Chem. 44, 149 (1940).

(Paper 65C1-51). 